\title{
BMJ Open Minimally invasive endoscopic staging for mediastinal lymphadenopathy in lung cancer: a systematic review protocol
}

\author{
Hong Liu, ${ }^{1}$ Jie Zhou, ${ }^{2}$ Qiao-ling Feng, ${ }^{3}$ Gang Wan, ${ }^{4}$ Yong-jun Xie, ${ }^{5,6}$ Hai-tao Gu ${ }^{1}$
}

To cite: Liu H, Zhou J, Feng Q-ling, et al. Minimally invasive endoscopic staging for mediastinal lymphadenopathy in lung cancer: a systematic review protocol. BMJ Open 2014;4: e005707. doi:10.1136/ bmjopen-2014-005707

- Prepublication history for this paper is available online. To view these files please visit the journal online (http://dx.doi.org/10.1136/ bmjopen-2014-005707).

Received 15 May 2014 Accepted 11 July 2014

CrossMark

For numbered affiliations see end of article.

Correspondence to Dr Jie Zhou;

zhoujiegrm@sina.com

\section{ABSTRACT}

Introduction: Minimally invasive endoscopic biopsy techniques have been widely available as potential alternatives for mediastinal lesions staging in patients with known or suspected lung cancer. Previous efforts have been made to evaluate the diagnostic performance of specific endoscopic modality alone at the level of the mediastinum for staging lung cancer, however, few studies focus on the accuracy of comparisons between different endoscopic modalities, especially at the level of any individual lymph node station. The objective of our study is to determine the diagnostic yields of different endoscopic modalities for staging mediastinal lymphadenopathy in lung cancer, especially concerning the individual lymph node station.

Methods/design: A systematic electronic search of MEDLINE, EMBASE, SinoMed and ISI Web of Science were performed to identify studies evaluating endoscopic modalities accuracy with restriction of English and Chinese languages from inception to an update until May 2014. Data were extracted with the patient as the unit of analysis with regards to the abilities of different endoscopic modalities at the level of mediastinum and particular lymph node station. The methodological quality was assessed independently according to the Quality Assessment of Diagnostic Accuracy Study (QADAS) criteria. An exact binomial rendition of bivariate mixed-effects regression model was used to estimate the pooled sensitivity and specificity. Also, pre-post probability analysis, publication bias analysis and sensitivity analysis were performed for a synthesis of knowledge of this context.

Dissemination: The findings will advance our better available knowledge of optimal clinical decision-making when dealing with staging of mediastinal metastasis in lung cancer.

Trial registration number: PROSPERO-NIHR Prospective Register of Systematic Reviews (CRD42014009792).

\section{INTRODUCTION}

Lung cancer has the highest morbidity among all cancers, with an estimated

\section{Strengths and limitations of this study}

- The systematic review is focused on different minimally invasive endoscopic techniques designed for use in the setting of lung cancer.

- The systematic review is non-commercial and has been elaborated systematically by a multidisciplinary panel of experts, working on behalf of the key stakeholders within a nationalised healthcare system.

- Any single endoscopic regime cannot be recommended as a definitive test for staging mediastinal lymphadenopathy in lung cancer, but rather it must be interpreted in context of the demographic and clinical factors, if feasible.

incidence of over 1.6 million cases/year accounting for $13 \%$ of all new cancer diagnoses; it is also the leading cause of cancerrelated deaths worldwide, with an estimated mortality of over 1.4 million/year, accounting for $18 \%$ of all cancer deaths. ${ }^{1}{ }^{2}$

Of crucial importance is accurate diagnosis and precise staging of known or suspected lung cancer for the clinician to better determine treatment, guide prognosis and facilitate continued investigation. ${ }^{3}$ Central to the diagnostic algorithm is the pathological staging in which the evaluation of mediastinal lymph node is a key step for the management of patients with lung cancer, especially in the absence of distant metastases. $^{45}$

Non-invasive imaging scans involving CT, positron emission tomography (PET) and the integrated PET/CT, ${ }^{6}{ }^{7}$ are considered favourable for staging mediastinal lymph nodes due to the morphological and functional characteristics of the lesions. ${ }^{8} 9$ However, more precise information on staging and typing is required for clinical decision-making. ${ }^{10}$

Mediastinoscopy and thoracoscopy have been recommended as diagnostic standards 
for staging along with histopathological validation of suspected mediastinal lymph nodes involvements. ${ }^{11}{ }^{12}$ Considering the extensive invasiveness and general anaesthesia, these tests are likely at a high risk of procedure-related complications with a reported incidence rate in the order of $2-3 \% .{ }^{13}$ Conversely, the invasive nature has led to an increasing interest in the development of more technically flexible, efficient and minimally invasive modalities coupled with comparable diagnostic yields.

With the advent of endoscopic biopsy techniques, the diagnostic algorithm for staging lung cancer has evolved into the minimally invasive stage. ${ }^{14}{ }^{15}$ Ideally, endobronchial ultrasound (EBUS) and endoscopic ultrasound (EUS) to guide transbronchial needle aspiration (TBNA) have been suggested for diagnosing and staging of mediastinal lesions in lung cancer. ${ }^{16-18}$ These endoscopic techniques have the significant advantages of being less invasive and with fewer complications, and have the potential to be reasonable alternatives to invasive staging modalities in certain populations, ${ }^{19-21}$ in addition to those of cytopathological phenotyping as well as molecular profiling for genotyping of lung cancer over non-invasive modalities. ${ }^{22}$

Previous efforts have been made to evaluate the diagnostic performance of specific endoscopic modality alone at the level of the mediastinum for staging lung cancer, ${ }^{23-30}$ however, few studies focus on the accuracy of comparisons between different endoscopic modalities, especially at the level of any individual lymph node station. Also, they call into question the possible association of the demographic and clinical confounders with the diagnostic accuracy of endoscopic modalities for diagnosis and staging of lung cancer. ${ }^{31}$

Given that a considerable need for a comprehensive systematic review has been triggered to synthesise the currently available bulk of information, the objective of this study is to ascertain the diagnostic yields of different minimally invasive endoscopic modalities for staging mediastinal lymphadenopathy in lung cancer, especially concerning the individual lymph node station.

\section{METHODS}

\section{Conception and design}

The design of this systematic review was elaborated by the multidisciplinary efforts (eg, cardiothoracic surgery, ultrasonography, radiation oncology, diagnostic medicine and health statistics) using methodological approaches outlined in the Cochrane Handbook for Systematic Review of Diagnostic Test Accuracy. ${ }^{32}$ This protocol refers to the Preferred Reporting Items for Systematic reviews and Meta-Analyses (PRISMA) criteria. ${ }^{33}$ This systematic review has been registered with No. CRD42014009792 in the Centre of Review and Dissemination (CRD) of York University, PROSPERO (the National Institute for Health Research (NIHR) International Prospective Register of Systematic Reviews).
Eligibility criteria for considering studies

Eligible articles will be identified in accordance with the PICOS criteria ${ }^{33}$ : Participants (pretreatment patients with suspected or previously diagnosed lung cancer for staging of mediastinal lymph nodes); Interventions (minimally invasive endoscopic techniques: TBNA, EUS-TBNA and EBUS-TBNA); Comparisons (histopathological validation following mediastinoscopy and surgery or close clinical follow-up for at least 6 months); Outcomes (diagnostic sensitivity, specificity, ORs and likelihood ratio) and Study design (any prospective cohort and case-control study).

\section{Search for identification of studies}

In addition to Cochrane Central databases (including the Cochrane Database of Systematic Reviews, the Cochrane Central Register of Controlled Trials, the Cochrane Methodology Register, the Database of Abstracts of Reviews of Effects, the Health Technology Assessment Database and the National Health Service (NHS) Economic Evaluation Database), a systematic electronic literature search of MEDLINE, EMBASE, SinoMed and ISI Web of Science will be performed to identify studies that have evaluated the accuracy of endoscopic techniques compared with reference standards in pretreatment patients with suspected or known lung cancer for staging of mediastinal lymph nodes; these studies will be eligible for inclusion. Search will be limited to articles published in English and Chinese, from inception to an update until May 2014. BIOSIS will be used to identify relevant abstracts and conference proceedings, and abstracts and conference proceedings will be included if appropriate. A combination of subject headings and text words will be used for search on the basis of three sets of terms: index tests (minimally invasive techniques aforementioned), target condition (suspected or known lung cancer for staging of mediastinal lymph nodes) and participants description (pretreatment patients with suspected or known lung cancer). Through the process of search, no filter will be used for diagnostic studies to maximise the sensitivity of the search strategies. Further relevant articles will be retrieved for recall completion by searching the bibliography of identified trials as well as other related systematic and narrative reviews.

\section{Search strategy}

Specific search strategy will be developed for each electronic database, commencing with MEDLINE (table 1). The MEDLINE strategy will be adapted for each subsequent database and search yields reported and compared between databases.

\section{Study screening}

The primary, subsequent and conclusive screening for inclusion will be on the basis of the article title, abstract and full text, respectively. The screening at each step will adhere to the same inclusion and exclusion criteria. At 
Table 1 Searching strategies of the Medline database (results from 14 May 2014)

\begin{tabular}{|c|c|c|c|}
\hline Specified item & Search & Query & Result \\
\hline \multirow{5}{*}{$\begin{array}{l}\text { Target condition } \\
\text { set }\end{array}$} & $\# 1$ & "Lung Neoplasms"[Mesh] & 170510 \\
\hline & \#2 & ("lung"[tw] OR pulmonary[tw]) & 924000 \\
\hline & \#3 & $\begin{array}{l}\text { (neoplasm* }\left[\text { tw] OR cancer* }{ }^{*} \text { tw] OR tumor* }[\text { tw] OR carcinoma*[tw] OR neoplasia* }\right. \\
\text { [tw] OR malignan*[tw]) }\end{array}$ & 2207899 \\
\hline & \#4 & "neoplasm staging"[MeSH Terms] & 119312 \\
\hline & \#5 & Stage[tw] OR stages[tw] OR staging[tw] & 806735 \\
\hline $\begin{array}{l}\text { Patient } \\
\text { description set }\end{array}$ & \#6 & ("humans"[MeSH] OR human*[tw]) NOT ("animals"[MeSH] OR animal*[tw]) & 11775100 \\
\hline \multirow[t]{15}{*}{ Index tests set } & $\# 7$ & $\begin{array}{l}\text { ("endosonography"[MeSH] OR "ultrasonography, interventional" [MeSH]) AND } \\
\text { "biopsy"[MeSH] }\end{array}$ & 4989 \\
\hline & \#8 & "Endoscopic Ultrasound-Guided Fine Needle Aspiration”[MeSH] & 385 \\
\hline & $\# 9$ & biops $^{*}[\mathrm{tw}]$ OR aspirat* $\left[\right.$ tw] OR punct ${ }^{*}[\mathrm{tw}]$ & 498201 \\
\hline & \#10 & endobronch*[tw] OR transbronch*[tw] OR (intervention*[tw] AND bronch*[tw]) & 12851 \\
\hline & \#11 & endosonograph*[tw] & 9756 \\
\hline & \#12 & Endoscop*[tw] & 169758 \\
\hline & \#13 & $\begin{array}{l}\text { Ultrasonography[MeSH] OR ultrasound*[tw] OR *sonogra*[tw] OR *ultrason*[tw] } \\
\text { OR echograph*[tw] OR Echo[tw] }\end{array}$ & 490594 \\
\hline & \#14 & \#1 OR (\#2 AND \#3) & 224785 \\
\hline & \#15 & (\#3 AND \#5) OR \#4 & 119312 \\
\hline & \#16 & \#14 AND \#15 & 16683 \\
\hline & $\# 17$ & \#6 AND \#16 & 16406 \\
\hline & $\# 18$ & \#9 AND \#10 AND \#11 & 405 \\
\hline & $\# 19$ & \#9 AND \#10 AND \#12 AND \#13 & 291 \\
\hline & \#20 & \#7 OR \#8 OR \#18 OR \#19 & 5133 \\
\hline & \#21 & \#17 AND \#20 & 364 \\
\hline
\end{tabular}

the first step, a single author will exclude studies clearly irrelevant to lung cancer or endoscopic staging for mediastinal lymphadenopathy in terms of the abstracts and titles. In case of selection bias, a $10 \%$ random sample of all potential references will be validated by a second author for agreement. A further screening will be conducted by at least two independent reviewers for each article reserved after the first step. Definite inclusion decision will be made on the basis of full text of relevant reports by two independent reviewers. Disagreements will be resolved by discussion and consultation with the aid of a third reviewer, if required.

\section{Inclusion criteria}

Population and target condition: The study population was patients either suspected or with known lung cancer who received endoscopic staging for mediastinal lymphadenopathy.

Index tests and reference standard: Patients were staged based on at least one minimally invasive endoscopic technique (eg, TBNA, EUS-TBNA and EBUS-TBNA), which should be compared with at least one of the reference standards (eg, tissue histological confirmation of mediastinoscopy and surgery or close clinical follow-up for at least 6 months), irrespective of availability of imaging-based staging before endoscopic techniques.

\section{Exclusion criteria}

Studies focusing especially on mediastinal node staging alone; studies consisting of patients with primary underlying disease other than lung cancer; studies unable to populate two-by-two contingency tables of test performance (absolute numbers of true-positive, false-negative, false-positive and true-negative results) from the text, appendices or despite contacting the authors; studies of sample sizes of less than 15 patients; studies of restaging after induction therapy; studies where the positive results from index tests were scarcely confirmed by any reference standard; and studies where duplicates or subcohorts have already been published. At least two independent reviewers will assess the papers for inclusion and exclusion criteria.

\section{Data extraction}

The clinical, demographic and methodological quality characteristics of the reference and index tests, as well as the diagnostic results (eg, true positives, true negatives, false positives and false negatives), will be extracted independently by two reviews. An in-depth discussion of the variability between studies will be provided where applicable. If possible, data for endoscopic biopsies performed at hilar, subcarinal, paratracheal or other lymph node stations will be extracted separately. Additional data were requested from original study investigators, if needed. In 
addition, the publication year, the study period, setting (eg, community vs academic hospitals; and primary vs tertiary centres), design (eg, cohort or case-control; and consecutive or random series), centre (eg, single or multiple), potential differences in reference standards, follow-up period and prevalence of mediastinal metastases will be described as covariates that may be the cause of heterogeneity. Two reviewers will independently extract data. Any disagreements will be resolved by consensus.

\section{Assessment of methodological quality}

In terms of the risk of bias and applicability concerns, the methodological quality of the articles included will be independently assessed by two reviewers using a subset of criteria derived from the Quality Assessment of Diagnostic Accuracy Study 2 (QUADAS-2) tool. ${ }^{34}$ A third reviewer will be consulted in case of discrepancies. Blinding implies that the results of minimally invasive endoscopic staging results must be interpreted without the knowledge of those of reference standards. Reviewers' judgments about risks of bias and applicability concerns will be used in sensitivity analysis to determinate the potential effect of methodological quality on diagnostic performance. Two reviewers will independently assess study quality. Any disagreements will be resolved by consensus.

\section{Data synthesis and analysis}

For each study we will collect the rates of true positives, false positives, true negatives and false negatives and produce from the crude data the sensitivity and specificity. In addition to the diagnostic accuracy of different endoscopic modalities for staging total mediastinal lymphadenopathy, we will shed some light on the accuracy for individual lymph node station and the lesions in different compartments of the mediastinum. A bivariate mixed-effects regression model will be used to generate the pooled estimates of sensitivity and specificity when appropriate. ${ }^{35}$ Meanwhile, summary receiver operating characteristic (SROC) curves with the corresponding area under the curve (AUC) will also be graphically generated to determinate the diagnostic accuracy, in which a summary operating point (pooled sensitivity and specificity) with the corresponding $95 \%$ CI and $95 \%$ prediction region, and the point $\mathrm{Q}^{*}$ (maximum joint sensitivity and specificity) will also be calculated in addition to the point estimates for each study as well as a symmetrical summary curve. Comparison of diagnostic accuracy between the groups will be achieved by $Z$ test on the basis of AUC of SROC.

\section{Threshold analysis}

Spearman's rank correlation will be performed for exploring the effect of threshold effect. To ensure the effect of variability in diagnostic threshold on the shape of SROC curve, threshold effect will be detected by the regression equation $\mathrm{D}=\mathrm{a}+\mathrm{bS}$, where $\mathrm{D}$ is the $\log$ of the diagnostic $\mathrm{OR}$ and $\mathrm{S}$ is a measure of the diagnostic threshold. ${ }^{36} 37$ The variables $\mathrm{a}$ and $\mathrm{b}$ will then be estimated using a least-squares method weighted by inverse variance.

\section{Heterogeneity investigation}

The expected heterogeneity across studies will be detected by the meta-regression analysis and subgroup analysis. The pattern of heterogeneity will be detected using $\chi^{2}$ test and the magnitude using $\mathrm{I}^{2}$ statistic. A study with an $\mathrm{I}^{2}$ greater than $50 \%$ will be considered substantially heterogenous. ${ }^{38}$

\section{Meta-regression analysis}

Meta-regression will be performed to evaluate the importance of potential effect variables and explain variation between studies. Univariate analysis will allow the investigation of the potential source of statistical heterogeneity. Subsequently, multivariate analysis will allow the determination of the important confounders affecting the diagnostic performance by the method of backward elimination. The covariates are consisted with the demographic, clinical and endoscopic characteristics.

\section{Subgroup analysis}

To explore clinical heterogeneity, we will fit a separate SROC curve for these planned subgroups of patients: (1) different anatomic compartments of the mediastinum (superior vs middle vs inferior mediastinum); (2) different types of endoscopic techniques (TBNA vs EUS-TBNA vs EBUS-TBNA vs incorporation of the modalities); (3) different populations (known vs suspected mediastinal lymph node involvement; with vs without the distant metastasis) and (4) sequential imaging (with vs without imaging scans before the implement of endoscopic techniques.

\section{Probability analysis}

The pretest probabilities of different prevalences of mediastinal metastases among patients suffering from lung cancer and corresponding post-test probabilities will be evaluated, depending on the summary sensitivity and specificity by Fagan's analysis, ${ }^{39}$ which will allow the determination of the relationship between the pretest and post-test probability as well as the likelihood ratio. The difference in the proportions of mediastinal lymphadenopathy in lung cancer (post-test probability minus pretest probability) will gave the overall added value of endoscopic staging.

\section{Publication bias}

Publication bias will be examined visually by inspecting funnel plots and statistically by using Egger's regression model. ${ }^{40}$ If publication bias was present, the effect of such bias on the summary estimate would be assessed using the trim and fill method. This method will impute the missing studies and recalculate a new summary estimate. $^{41}$ The difference between the calculated and 
observed value will be then used to determine the effect of bias on the diagnostic performance of the index tests.

\section{Post-hoc power analysis}

The technique of power analysis allows for the determination of how likely a statistical test of an individual study will be to detect effects of a given sample size in a particular situation. The determination of post-hoc power for testing differences in proportions for the matched pair design with binary response outcome will be performed for each study included, according to the formula derived by Lachin. ${ }^{42}$

\section{Sensitivity analysis}

For detecting the robustness of pooled results, sensitivity analysis will be performed to determine the potential impacts of study design on endoscopic staging accuracy. ${ }^{43}$ On the basis of QUADAS-2, the important following sensitivity analyses will be prespecified: risk of selection bias, risk of interpretation bias for the index tests as well as reference standards. ${ }^{34}$

All the analyses will be conducted using MADIS module of Stata V.10 (Stata Corp, College Station, Texas, USA), except for publication bias using Meta-Analyst $\beta 3.13$ (Tufts Medical Center, Boston, Massachusetts, USA), and meta-regression and threshold analysis using Meta-DiSc V.1.4 (Clinical Biostatistics Unit, Ramón y Cajal Hospital, Madrid, Spain).

\section{DISCUSSION}

The problem-oriented research will enable the best available knowledge of clinical practice, on the strength of comprehensively rigorous methodology used in the review. The internal validation and navigation involved in the process will minimise the potential of selection bias and systematic errors. On the basis of a synthesis of methodological quality estimation for each study included, the findings will be available for a further subgroup analysis to explore potential methodological heterogeneity. Considering the high dependence of the findings on the quality of the underlying primary studies, as well as the potential risk of bias from limited quality studies on clinical practice, only the prospective studies will be included in our current review in order to provide more convincing evidence.

The findings of this systematic review will provide important evidence for endoscopic staging for mediastinal lymphadenopathy in lung cancer, as well as enable optimal clinical decision-making when involved in staging for mediastinal lymph node involvement. The findings will also help to better advance our available knowledge of the management strategy in individualised, targeted and comprehensive treatment of, as well as aiding in the determination of prognosis among, patients suffering from lung cancer. Furthermore, the findings may trigger an update or drive the development of related standards, which will lead to refine mediastinal staging in lung cancer.
To our knowledge, the current review will allow the diagnostic accuracy of different endoscopic staging for mediastinal lymphadenopathy in lung cancer, for the first time, from an overall level to the individual node station level, on the basis of evidence-based research. Given that we focus on results from practice and emphasise outcomes evaluation, the results from this review will put forth the advantages and disadvantages in medical practice of using minimally invasive endoscopic biopsy in staging mediastinal lymphadenopathy in lung cancer, and will lay the foundation for further research and the development of establishing optimal staging pathways for patient subgroups.

\section{Author affiliations}

${ }^{1}$ Department of Cardiothoracic Surgery, First Affiliated Hospital of Nanjing Medical University, Nanjing, China

${ }^{2}$ Department of Ultrasonography, First Affiliated Hospital of Nanjing Medical University, Nanjing, China

${ }^{3}$ Key Laboratory of Diagnostic Medicine of Education Ministry, Institute of Laboratory Medicine, Chongqing Medical University, Chongqing, China ${ }^{4}$ Department of Radiation Oncology, Affiliated Cancer Hospital of Guangxi Medical University, Nanning, China

${ }^{5}$ National Center for Medical Simulation of China, Chengdu Medical College, Chengdu, China

${ }^{6}$ Department of Histo-anatomy, School of Basic Medical Sciences, Chengdu Medical College, Chengdu, China

Acknowledgements The authors thank CZ Chen, an information specialist of Dana-Farber Cancer Institute of Harvard Medical School, for his assistance with search strategy design, and XS Li, a senior statistician of Sichuan University Western China School of Medicine, China Center for Chinese Clinical Trial Register.

Contributors HL conceived and designed the review and completed the PROSPERO registration; $\mathrm{HL}$ and $\mathrm{JZ}$ conducted the scoping searches and drafted and revised the manuscript. GW and Q-IF were involved in the design of the review and piloted the inclusion and exclusion criteria and the extraction forms. HL, JZ, Q-IF, GW, H-tG and Y-jX provided content expertise and feedback on the design of the review, the protocol and on the manuscript. All authors shared the interpretation of data and critical revision of the manuscript for important intellectual content. All authors critically reviewed the first draft and contributed to the production of the final manuscript and its subsequent revision.

Funding Funded by Priority Academic Program Development (PAPD) of Jiangsu Higher Education Institutions, China (JX10231081).

Competing interests $\mathrm{JZ}$ is funded by the Priority Academic Program Development (PAPD) of Jiangsu Higher Education Institutions (JX10231081).

Patient consent Obtained.

Provenance and peer review Not commissioned; peer reviewed for ethical and funding approval prior to submission.

Open Access This is an Open Access article distributed in accordance with the Creative Commons Attribution Non Commercial (CC BY-NC 4.0) license, which permits others to distribute, remix, adapt, build upon this work noncommercially, and license their derivative works on different terms, provided the original work is properly cited and the use is non-commercial. See: http:// creativecommons.org/licenses/by-nc/4.0/

\section{REFERENCES}

1. Jemal A, Bray F, Center MM, et al. Global cancer statistics. CA Cancer J Clin 2011;61:69-90.

2. Lewis SZ, Diekemper R, Addrizzo-Harris DJ. Methodology for development of guidelines for lung cancer: diagnosis and management of lung cancer, 3rd ed: American College of Chest 
Physicians evidence-based clinical practice guide-lines. Chest 2013;143(Suppl):41S-50S.

3. Vanderlaan PA, Wang $\mathrm{HH}$, Maiid A, et al. Endobronchial ultrasound-guided transbronchial needle aspiration (EBUS-TBNA): an overview and update for the cytopathologist. Cancer Cytopathol 2014 [epub ahead of print 23 Apr 2014]. doi:10.1002/cncy.21431

4. Cornwell LD, Bakaeen FG, Lan CK, et al. Endobronchial ultrasonography-guided transbronchial needle aspiration biopsy for preoperative nodal staging of lung cancer in a veteran population. JAMA Surg 2013;148:1024-9.

5. Annema JT, Versteegh MI, Veseliç M, et al. Endoscopic ultrasound added to mediastinoscopy for preoperative staging of patients with lung cancer. JAMA 2005;294:931-6.

6. Perigaud C, Bridji B, Roussel JC, et al. Prospective preoperative mediastinal lymph node staging by integrated positron emission tomography-computerised tomography in patients with non-small-cell lung cancer. Eur J Cardiothorac Surg 2009;36:731-6.

7. Maziak DE, Darling GE, Inculet RI, et al. Positron emission tomography in staging early lung cancer: a randomized trial. Ann Intern Med 2009;151:221-8.

8. Kramer H, Groen HJM. Current concepts in the mediastinal lymph node staging of nonsmall cell lung cancer. Ann Surg 2003:238:180-8.

9. Pieterman RM, van Putten JWG, Meuzelaar JJ, et al. Preoperative staging of non-small cell lung cancer with positron emission tomography. N Engl J Med 2000;343:254-61.

10. De Leyn P, Lardinois D, Van Schil PE, et al. ESTS guidelines for preoperative lymph node staging for nonsmall cell lung cancer. Eur $J$ Cardiothorac Surg 2007;32:1-8.

11. Rusch VW. Mediastinoscopy: an endangered species? J Clin Oncol 2005;23:8283-5.

12. Dhaliwal CA, Andrews TD, Walker WS, et al. Histological evaluation of preoperative mediastinoscopy lymph node biopsies in non-small cell lung cancer. J Clin Pathol 2014;67:76-80.

13. Hammond ZT, Anderson RC, Meyers BF. The current role of mediastinoscopy in the evaluation of thoracic disease. $J$ Thorac Cardiovasc Surg 1999;118:894-9.

14. Herth F. Nonsurgical staging of the mediastinum: EBUS and EUS. Semin Respir Crit Care Med 2011;32:62-8.

15. Becker HD. EBUS: a new dimension in bronchoscopy. Of sounds and images-a paradigm of innovation. Respiration 2006;73:583-6.

16. Herth FJ, Eberhardt R. Actual role of endobronchial ultrasound (EBUS). Eur Radiol 2007;17:1806-12.

17. Cetinkaya E, Gunluoglu G, Ozgul A, et al. Value of real-time endobronchial ultrasound-guided transbronchial needle aspiration. Ann Thorac Med 2011;6:77-81.

18. Ost DE, Ernst A, Lei $X$, et al. AQuIRE Bronchoscopy Registry. Diagnostic yield of endobronchial ultrasound-guided transbronchial needle aspiration: results of the AQuIRE Bronchoscopy Registry. Chest 2011:140:1557-66.

19. Vilmann P, Krasnik M, Larsen SS, et al. Transesophageal endoscopic ultrasound-guided fine-needle aspiration (EUS-FNA) and endobronchial ultrasound-guided transbronchial needle aspiration (EBUS-TBNA) biopsy: a combined approach in the evaluation of mediastinal lesions. Endoscopy 2005;37:833-9.

20. Yasufuku K, Nakajima T, Waddell T, et al. Endobronchial ultrasound-guided transbronchial needle aspiration for differentiating N0 versus N1 lung cancer. Ann Thorac Surg 2013;96:1756-60.

21. Leong S, Shaipanich T, Lam S, et al. Diagnostic bronchoscopycurrent and future perspectives. $J$ Thorac Dis 2013;5(Suppl 5): S498-510.

22. Bhutani MS, Jones DV Jr, Zwischenberger JB. Endoscopic ultrasound-guided fine-needle aspiration staging of lung cancer: is it time to go beyond cytology? Chest 2005;127:418-20.

23. Dong X, Qiu X, Liu Q, et al. Endobronchial ultrasound-guided transbronchial needle aspiration in the mediastinal staging of non-small cell lung cancer: a meta-analysis. Ann Thorac Surg 2013;96:1502-27.
24. Adams K, Shah PL, Edmonds L, et al. Test performance of endobronchial ultrasound and transbronchial needle aspiration biopsy for mediastinal staging in patients with lung cancer: systematic review and meta-analysis. Thorax 2009;64: 757-62.

25. Tournoy KG, Keller SM, Annema JT. Mediastinal staging of lung cancer: novel concepts. Lancet Oncol 2012;13:e221-9.

26. Gu P, Zhao YZ, Jiang LY, et al. Endobronchial ultrasound-guided transbronchial needle aspiration for staging of lung cancer: a systematic review and meta-analysis. Eur J Cancer 2009;45:1389-96.

27. Varela-Lema L, Fernández-Villar A, Ruano-Ravina A. Effectiveness and safety of endobronchial ultrasound-transbronchial needle aspiration: a systematic review. Eur Respir J 2009;33: 1156-64.

28. Zhu T, Zhang $\mathrm{X}, \mathrm{Xu} \mathrm{J}$, et al. Endobronchial ultrasound guided-transbronchial needle aspiration vs. conventional transbronchial needle aspiration in the diagnosis of mediastinal masses: a meta-analysis. Mol Clin Oncol 2014;2:151-5.

29. Zhang R, Ying K, Shi L, et al. Combined endobronchial and endoscopic ultrasound-guided fine needle aspiration for mediastinal lymph node staging of lung cancer: a meta-analysis. Eur J Cancer 2013;49:1860-7.

30. Wang Memoli JS, Nietert PJ, et al. Meta-analysis of guided bronchoscopy for the evaluation of the pulmonary nodule. Chest 2012;142:385-93.

31. Bhutani MS, Hofstetter W. Transesophageal EUS and EUS-FNA for lung cancer: where do we go from here? J Clin Gastroenterol 2007;41:644-6.

32. Deeks JJ, Bossuyt PM, Gatsonis C. Cochrane handbook for systematic reviews of diagnostic test accuracy version 1.0.0. The Cochrane Collaboration, 2008. http://srdta.cochrane.org/ handbook-dta-reviews

33. Liberati A, Altman DG, Tetzlaff $\mathrm{J}$, et al. The PRISMA statement for reporting systematic reviews and meta-analyses of studies that evaluate health care interventions: explanation and elaboration. $B M$ 2009;339:b2700.

34. Whiting PF, Rutjes AW, Westwood ME, et al. QUADAS-2: a revised tool for the quality assessment of diagnostic accuracy studies. Ann Intern Med 2011;155:529-36.

35. Johannes BR, Afina SG, Anne WS, et al. Bivariate analysis of sensitivity and specificity produces informative summary measures in diagnostic reviews. J Clin Epidermiol 2005;58:982-90.

36. Moses L, Littenberg B, Shapiro D. Combining independent studies of a diagnostic test into a summary ROC curve: data-analytical approaches and some additional consideration. Stat Med 1993:12:1293-316.

37. Zamora J, Abraira V, Muriel A, et al. Meta-DiSc: a software for meta-analysis of test accuracy data. BMC Med Res Methodol 2006;6:31.

38. Schuetz GM, Schlattmann P, Achenbach S, et al. Individual patient data meta-analysis for the clinical assessment of coronary computed tomography angiography: protocol of the Collaborative Meta-Analysis of Cardiac CT (CoMe-CCT). Syst Rev 2013;2:13.

39. Hellmich $\mathrm{M}$, Lehmacher W. A ruler for interpreting diagnostic test results. Methods Inf Med 2005;44:124-6.

40. Egger M, Davey S, Schneider M, et al. Bias in meta-analysis detected by a simple, graphical test. BMJ 1997;315:629-35.

41. Duval S, Tweedie R. Trim and fill: a simple funnel-plot-based method of testing and adjusting for publication bias in meta-analysis. Biometrics 2000;56:455-63.

42. Lachin JM. Introduction to sample size determination and power analysis for clinical trials. Control Clin Trials 1981;2:93-114.

43. Macaskill P, Gatsonis C, Deeks J, et al. Analysing and presenting results. In: Deeks JJ, Bossuyt PM, Gatsonis C, eds. Cochrane handbook of systematic reviews of diagnostic test accuracy version 1.0.0. The Cochrane Collaboration, 2010:1-61. http://srdta.cochrane. org/ 